\title{
E-Governance: Herausforderungen und Chancen für Demokratie, Verwaltung und Recht
}

\author{
Bericht über den 26. Internationalen Kongress der Verwaltungswissenschaften \\ vom 14. bis 18. Juli 2004 in Seoul, Südkorea
}

Die Verwaltungsmodernisierung bewegt ihren Fokus in Forschung und administrativer Umsetzung vom lange beherrschenden Thema E-Government hin zu E-Governance, das als das umfassendere, also auch als das EGovernment einschließende Thema angesehen wird. Je umfassender aber die E-Verwaltungsmodernisierung unter dem Aspekt der »E-Governance angegangen wird, um so mehr entstehen gleichzeitig Herausforderungen und Chancen für die Demokratie, die Verwaltung und das Recht.

Zugleich ist E-Governance ein weltweiter Trend in öffentlichen Verwaltungen, der in vielen Ländern zeitgleich aufgegriffen wird und zugleich zu einem Richtungswechsel veranlasst. Er führt zu neuen Wegen des Regierens und Verwaltens, unter Einbeziehung und gegenseitiger Befruchtung des öffentlichen und des privaten Sektors. Solch ein internationaler Trend bedarf einer internationalen wissenschaftlichen Aufarbeitung. Hier war der Kongress in der südkoreanischen Hauptstadt ein Meilenstein.

Die Konferenz des seit 1930 bestehende Internationalen Instituts für Verwaltungswissenschaften mit Sitz in Brüssel ${ }^{1}$ (IISA/IIAS) fand wieder als Doppelveranstaltung zusammen mit der Jahreskonferenz der Internationalen Vereinigung der Verwaltungsschulen und Verwaltungsinstitute (IASIA/AIEIA) statt. Seoul im Juli verlangte den mehr als 300 registrierten Teilnehmern und ca. 100 Referenten allerdings einige klimatische Anpassungsfähigkeit $\mathrm{ab}$. Grund sind alljährlich im Sommer zusammentreffende Wärme, hohe Luftfeuchtigkeit und Jahreshöchstmengen an Monsunniederschlägen. Im wohlklimatisierten Konferenzzentrum COEX, das im Wirtschaftszentrum Gangnam-gu der 12-Millionen-Metropole liegt, und dank des beeindruckenden Engagements von Organisatoren, Referenten und Teilnehmern machte sich dies allerdings nicht negativ bemerkbar.

Die Bedeutung, die dem Thema E-Governance derzeit weltweit zukommt, wurde bereits anlässlich der feierlichen Eröffnung der Konferenz und anlässlich des Eröffnungsempfangs deutlich, als kein geringerer als Roh Moo-hyun, Präsident der Republik Korea, Gäste und Konferenzteilnehmer willkommen hieß. Der Präsident stellte den Wandel der Republik Korea von einem autoritär geführten Staat zu einer demokratischen Zivilgesellschaft heraus. Eine autoritär geführte Regierung und Verwaltung könne die durch Innovationen gesteuerte Wirtschaftsentwicklung in einer modernen Gesellschaft nicht garantieren. Nur mit Innovationsfreude auch im öffentlichen Sektor seien Wettbewerbsfähigkeit der Märkte und der Nationen zu sichern. Der Präsident verband außerdem Verwaltungsmodernisierung mit der unausweichlichen Notwendigkeit des lebenslangen Lernens und der aufgabenkritischen Selbstbeobachtung des eigenen Handelns aller Staatsbediensteten. In diesem innovationsgesteuerten Wandel in Regierung und Verwaltung erkannte er den Richtungswechsel der Governance.

Der Generalberichterstatter der Konferenz, Pan Suk Kim aus Korea, wies eingangs auf die immens gesteigerten Interaktions- und Kommunikationsmöglichkeiten für die Bürger untereinander oder mit Regierung und Verwaltung hin, welche die Verbreitung der Informations- und Kommunikationstechnik eröffne (»universe computing «). Die Welt sei dadurch noch kleiner geworden. Darüber hinaus habe E-Government ein ungeheueres Innovationspotential weltweit freigesetzt. E-Government biete allen Ländern neue Entwicklungschancen und sie seien gewillt, diese auch zu nutzen. Jedes Staatswesen könne aber seine Legitimation nur auf den Konsens der eigenen Bevölkerung gründen. E-Governance sei daher der Weg, zu einer gemeinsamen Politikgestaltung auf Grundlage dieses Bürgerkonsenses zu gelangen. Dies erfordere den befruchtenden Dialog mit den Regierenden mit dem Ziel einer umfassenden (Bürger-)Beteiligung am politischen und administrativen Prozess. E-Governance beschreibe also die Organisation und den Gebrauch politischer Macht.

E-Govenment richtete bisher den Fokus auf die Bereitstellung von Informationen und Transaktionen durch die Verwaltung für ihre Kunden intern oder extern, beleuchtete also nur einen Ausschnitt aller Zusammenhänge im Komplex Regieren und Verwalten. E-Governance dagegen möchte, gleichsam als übergeordnetes Thema, die Öffentlichkeit als Ganzes in ihrer Rolle als Bürger neu erfassen und unter Berücksichtigung aller Möglichkeiten neuer Informations- und Kommunikationstechniken (Rollen-) Veränderungen und Veränderungsmöglichkeiten im Zusammenhang mit Regieren und Verwalten aufzeigen. »Wer regiert eigentlich? «, könnte nämlich eine Frage am Ende eines gesellschaftlichen Informatisierungs- und Beteiligungsprozesses lauten, nachdem z.B. auch E-Democracy - ein weiterer nachgeordneter Themenkomplex neben E-Government - erfolgreich fortgeschritten ist. Eine andere zentrale Frage wäre: Führt E-Governance überhaupt zu »Good Governance «?
Diese Fragestellungen im Blick beleuchtete der Kongress E-Governance in vier Arbeitsgruppen: 1. Auswirkungen von E-Governance auf Zivilgesellschaft, Transparenz und Demokratie (Berichterstatter: Olli Mäenpää, Finnland), 2. Veränderungen von Verwaltungsstrukturen und -prozessen durch E-Governance (Berichterstatter: Shunichi Furukawa, Japan), 3. Perspektiven für Schwellen- und Entwicklungsländer durch E-Governance (Berichterstatter: Gordon Draper, Jamaica) und 4.: mit E-Governance einhergehende Rechtsentwicklungen (Berichterstatter: Herbert Maisl, Frankreich). Diese Themen wurden in 25 Workshops vertieft. Hinzu kamen: ergänzende Foren der Koreaner zum Thema »Administrative Innovation by e-Government in Korea« und der Vereinten Nationen zum Thema »The World Public Sector Report 2003: E-government at the Crossroads«, ein gemeinsames Forum von IIAS und IASIA (»The Implications of «E-Governance» for Building Leadership«), ein offenes Forum »IT and Woman«, ein Forum der OECD zum Thema »E-Governance as Tool for Public Management Reform: a Perspective from OECD«, ein Forum »Technology and Public Administration: conditions for success in e-government development «, ein Asia Pacific-Forum »NGOs in E-Governance« sowie ein Forum Finnlands zum Thema »Parliaments and E-Governance«.

Auf Grund der geographischen Lage Seouls kam über ein Drittel der Kongressteilnehmer aus dem asiatischen Raum. Die größten Teilnehmergruppen stellten dabei China (mit Taiwan), Korea, Japan und Indien. Aus Europa kamen etwas weniger als ein weiteres Teilnehmerdrittel, wobei Finnland wegen des finnischen Forums die weitaus größte Gruppe stellte. Aus Deutschland waren vier Teilnehmer angereist - zwei davon zugleich auch als Referenten. Aus dem überseeischen angloamerikanischen Sprachraum (Australien, Kanada, USA) kam etwa ein Fünftel der Teilnehmer.

Die Konferenzthemen und Beiträge der Referenten waren auf Grund der Teilnehmerzusammensetzung aus deutscher verwaltungswissenschaftlicher Perspektive sicher unterschiedlich zu bewerten. Ein guter Teil der Themen, Referate und Diskussionsgrundlagen betraf Länder mit vergleichsweise anderen Problemschwerpunkten, Strukturen und Entwicklungsfortschritten in E-Government und E-Governance. So konnten zum Beispiel China, Indien, Indonesien und einige arabische und afrikanische Länder während des Treffens in Korea sicher den befruchtenden Austausch untereinander finden. Gleichzeitig ermöglichte Seoul aber auch den Austausch unter den amerikanischen, australischen, europäischen und den sehr engagierten und erfolgreichen koreanischen Wissenschaftlern.

Von den Beiträgen seien zunächst besonders die beiden deutschen Referate hervorgehoben. Die Ausführungen von

- Hans-Peter Bull, Non-technological Problems of E-Voting und

- Ralf-Eckhard Türke, eGovernance: An Integrated Framework to Promote Governance

hatten eine überaus positive Resonanz.

Darüber hinaus war eine ganze Reihe weiterer Beiträge für die europäische und die deutsche Diskussion von Interesse. Sie können im Internet abgerufen werden unter http://itstandard.co.kr/ iias/iias_paper/iias_ papers.htm.

Abschließend sei nochmals die perfekte Organisation des Kongresses durch die koreanischen Gastgeber betont. Es war der Eindruck zu gewinnen, diese sähen die klimatischen Bürden als selbst verantwortet an und wollten diese durch ihre Gastfreundschaft ausgleichen. Technische und konferenzbegleitende Betreuung, die Organisation des Gala Dinners und des Exkursionsprogramms in Seoul am Ende der Konferenz - überall standen freundliche und hilfsbereite Menschen mit Rat und Tat zur Seite und ließen die sprichwörtliche asiatische Gastfreundlichkeit für alle Teilnehmer erlebbar werden.

Hans-Peter Bull, Präsident der Deutschen Sektion des IIAS/IISA, lud am Ende der Konferenz alle Teilnehmer zur nächsten IIAS/IISA-Jahreskonferenz ein, die unter dem Thema »Public Administration and Private Enterprise - Co-operation, Competition and Regulation « (Generalberichterstatter Luiz Carlos Bresser-Pereira, Brasilien) vom 20. bis 24. September in Berlin stattfinden wird. ${ }^{2}$

\section{Regierungsrat z.A. Markus Städler, Mag. rer. publ., Bundesverwaltungsamt Köln}

1 Für nähere Informationen zum Institut und zur Konferenz vgl. http://www.iiasiisa.be oder http://www.kipa.re.kr/iias

2 Für nähere Informationen zur Konferenz 2005 vgl. im Internet: http://www.iias-berlin-2005.de 